\title{
Reflexiones propedéuticas sobre la política lingüística en Colombia: El caso del bilingüismo (Anteproyecto de investigación para postulación)
}

\author{
Miguel Angel Mahecha Bermudez \\ Programa de Lengua Castellana \\ Universidad Surcolombiana \\ miguelangel.mahecha@usco.edu.co
}

\section{Preámbulo}

La evaluación de las políticas lingüísticas -con sus correspondientes planificaciones-, la economía de lenguas y la glotopolítica entre otras tantas, constituyen el marco de referencia más adecuado para entender cómo la política lingüística nacional y sus respectivas consecuencias en materia de enseñanza de las lenguas pueden ser comparadas en términos de asignación de presupuestos y de distribución de recursos, en otra palabras, es hablar en términos de eficiencia, competitividad y prospectiva. Esta relación afectaría directamente la configuración por ejemplo, de los proyectos pedagógicos de la Facultades, el papel de la lengua extranjera que se impone, como es el caso del inglés no sólo frente a otras lenguas sino frente al español pues la argumentación que justifica su enseñanza/aprendizaje radica más en el hecho de los diferenciales salariales netos que provienen del manejo de esta lengua y el prestigio social de quien la maneja que de la consideración de aspectos lingüísticos que caracterizan la realidad social regional.

Las políticas lingüísticas se consideran a menudo como orientaciones o instrumentos a nivel estatal y casi siempre desde una perspectiva de organización de la defensa de una o varias lenguas nacionales/ regionales (política lingüística interna) y/o de expansión de dicha(s) lengua(s) (política lingüística externa). Remiten entonces a los esfuerzos públicos que pretenden modificar el estatus relativo y el uso de una o varias lenguas. En el contexto de las construcciones nacionales, la unificación lingüística como reto administrativo y simbólico se ha plasmado a veces en el monolingüismo y otras veces en el reparto de territorios; y la política lingüística explícita se interesa entonces esencialmente por la definición de las normas gramaticales oficiales de la lengua vehicular y por las condiciones de enseñanza de las lenguas minoritarias, dejando a lo implícito ocupar el espacio de un dejar hacer.

La irrupción del plurilingüismo como dato consubstancial de las sociedades humanas perturba esta representación de la acción sobre las lenguas. Los actores sociales usuarios y difusores prescriptores, son numerosos y variados, del individuo a los grupos organizados (administraciones, empresas, asociaciones), y el objeto se considera necesariamente plural en las relaciones instituidas entre las lenguas por el conjunto de las actividades sociales entre lo local y lo global.

La cuestión que se plantea cuando se habla de plurilingüismo y de sus componentes comunicativos y didácticos como la intercomprensión o la educación a las lenguas consiste en determinar si el desafío no reside en los fundamentos, el diseño y la ejecución de políticas interlingüísticas explícitas.

- Grandes orientaciones teóricas y prácticas en materia de política lingüística

- Epistemología del plurilingüismo como objeto de política lingüística

- Espacio del plurilingüismo en las políticas lingüísticas internas y externas

- Situaciones lingüísticas geopolíticas y políticas de plurilingüismo

- Relación entre políticas linguiísticas y didáctica

- La intercomprensión en las políticas lingüísticas

Lo que buscamos en el presente texto es esbozar una problemática a manera de invitación, con el propósito de evaluar la incipiente política lingüística colombiana y propender por una política lingüística integral, esto es, una política acorde con la realidad sociolingüística de Colombia. Para tal efecto, presentaremos en la primera arte un marco de referencia conceptual dentro del cual se inscribe la problemática; en segundo lugar, se llevará a cabo la reflexión utilizando como guía, el esquema argumentativo de Pardo/Baquero (1988). Se finalizará con la presentación de algunos anexos que ilustran las particularidades de la propuesta.

\section{Marco referencial}

La Sociolingüística: Sobre la sociolingüística se ha dicho tanto como se ha podido pero también se ha dejado de decir muchas cosas importantes. Lo que se mencionará aquí, será una serie de precisiones en 
cuanto a su base epistemológica y sus correspondientes proyecciones metodológicas.

Empecemos haciendo un recuento de orden histórico. Para ello nos apoyaremos en las declaraciones de los profesores Calvet (1993b y 2002), Marcellesi (1980), Marcellesi y Gardin (1974), José Pedro Rona (1974) y Coseriu (1981). Si bien la etiqueta "(socio)lingüística" se hace evidente en el encuentro de la UCLA (University of California Los Angeles) en $1964^{1}$ convocado por el profesor William Bright, la actividad sociolingüística como tal se he llevado a cabo mucho antes de esa época; basta con citar, a manera de ejemplo, el artículo de Antoine Meillet, Comment les mots changent de sens $^{2}$ para darse cuenta de este caso. En el discurso de apertura del coloquio de 1978 sobre la sociolingüística $^{3}$ J.B. Marcellesi hace una semblanza acertada de la configuración epistemológica del campo sociolingüístico:

La sociolinguistique n'a sans doute pas un objet rigoureusement défini et les épistémologues ont peut-être raison de faire la fine bouche. En tout cas l'une de nos finalités est d'arriver à une vue plus claire sur ces questions. Mais au vu de l'intérêt suscité un peu partout par ce colloque on peut dire que ceux qui seraient tentés de penser que la sociolinguistique n'existe pas doivent quand même s'interroger sur le caractère attractif de ce phantasme. Et sur l'existence en tout cas des sociolinguistes.

En el coloquio citado supra, se buscaba determinar si la sociolingüística era una visión diferente y renovada de la lingüística o por el contrario, no era más que un pleonasmo injustificado teóricamente. A lo largo del coloquio así como en la mesa redonda final ${ }^{4}$, diversos investigadores dieron su punto de vista, unos a favor del morfema 'socio-', es decir de su inclusión para completar lo lingüístico, otros en desacuerdo con él. No hay que olvidar que habían pasado ya catorce años desde el primer congreso de sociolingüística llevado a cabo en la UCLA en 1964, y a pesar de que su promotor, el profesor William Bright, proponía un objeto de estudio "definido" para este campo naciente, "La diversidad lingüística es precisamente el asunto principal de la sociolingüística" (Bright, 1974:198), otros autores como José Pedro Rona (1974:203) manifestaban lo contrario: "En ninguna ciencia es posible realizar trabajos de investigación adecuados si no se delimita previamente el objeto, la teoría y la metodología de la ciencia misma. Hasta donde sabemos, esto nunca fue hecho en el campo de la sociolingüística." (Destacado nuestro). Es más, si bien es cierto que la sociolingüística avanzó notablemente en la constitución de su método de trabajo, la claridad en cuanto a su objeto de estudio homogéneo no quedó clara. Exagerando un poco otra declaración de W. Bright, la sociolingüística era -y es- un cuerpo grande con una mente pequeña: "La sociolingüística es un niño en pleno desarrollo, pero que no deja de ser un niño."(1974:217). Por más esfuerzos que hayan hecho Labov, Bernstein, Hymes, Gumperz, Bright, Fishman y otros, el debate epistemológico mantiene su saldo en rojo. Los estudios sociolingüísticos, ya lo hemos dicho, son bastante heterogéneos, esto quiere decir que hay varios objetos y puntos de vista diferentes y la unificación es casi imposible de lograr. De ahí que valga la pena preguntarse si la sociolingüística puede lograr el estatus de disciplina autónoma, o por el contrario, es una etiqueta que disfraza el punto de encuentro de varias disciplinas ${ }^{5}$. Esta proliferación de puntos de vista ha llevado a que autores como Coseriu, indiquen tres tipos de estudios sociolingüísticos: "a) estudio

1 - Para detalles más precisos sobre este encuentro véase: William Bright ed., Sociolinguistics. Proceedings of the UCLA Sociolinguistics Conference (La Haye: Mouton, 1966).Christina Bratt Paulston y Richard Tucker eds., The early days of sociolinguistics. Memories and reflections (Summer Institute of Linguistics, 1997). Louis-Jean Calvet, « Aux origines de la sociolinguistique, la conférence de sociolinguistique de l'UCLA (1964) », Langage \&Société 88 , juin

2 - Artículo publicado en Linguistique historique et linguistique générale, Paris, Champion, 1965, p.230-271. De igual manera, Coseriu presenta otro ejemplo que ilustra este caso: "Fn cierto sentido, la tarea de la sociolingüística fue delineada ya en 1536 (j428 años antes del encuentro de la UCLA! MM) por el genial gramático portugués Fernâo de Oliveira, guiado en esto sólo por su robusto sentido común y por sus extraordinarios dones de observador de los hechos lingüísticos:" (1981:5).

3 - Las memorias de este encuentro fueron publicadas por Bernard Gardin et J.B. Marcellesi eds., Sociolinguistique. Approches, théories, Pratiques (Paris, PUF, tomes I et II., 1980). Traducimos aquí los datos de la presentación del coloquio realizados por la prensa local francesa: "El coloquio 'Teorías y prácticas de la sociolingüística' se inauguró el lunes 27 de noviembre en la Facultad de Letras de la Universidad de Rouen (Mont-SaintAignan). Los congresistas fueron recibidos por el decano de la facultad, M.A. Sadourny quien rindió un homenaje al equipo de lingüistas de la universidad sede por lograr mantener un sano equilibrio entre el ejercicio de la docencia y el de la investigación. En esta dirección, un evento como el coloquio de sociolingüística se inscribe en una política de sobrevivencia, y en lo posible, de desarrollo de la investigación universitaria en HauteNormandie. En ausencia del presidente de la universidad, M. J.M.Helbert saluda a su vez a los congresistas y les presenta las estructuras y las actividades de la universidad [...] M. Helbert se alegró tambiến por el interés mostrado hacia la sociolingüística, ciencia insertada en lo social, preocupada por la sociedad y sus estructuras y cuyo aporte es esencial para todo practicante de la enseñanza. Destaca al final, el carácter internacional del coloquio que agrupa a profesores e investigadores de 10 países entre los que figuran México y Canadá.

A partir del 27 de noviembre hacia las dos de la tarde, se dio inicio a las sesiones plenarias, las reuniones en talleres y las discusiones libres...Citemos a los presidentes de sesión: H. Greven (sesión sobre la sociolingüústica en los países extranjeros), W. Pollak (las situaciones de diglosia), J.-B. Marcellesi (lingüística y sociología), G. Jiménez (gramática y sociolingüística), F. François (sociolingüística y escuela), M. Tournier y J.L. Fossat (análisis del discurso), J. Peytard (sociolingüística del campo). Numerosos talleres y grupos de trabajo se llevaron a cabo fuera de las sesiones plenarias y dieron lugar a intercambios fructíferos: "sociología y lingüística" con P. Bourdieu, J.C. Chevalier y P. Encrevé, "el discurso de los intelectuales colectivos" con Bernard Gardin, "la sociolingüística italiana" con J.-B. Marcellesi, "Ia sociolingüística en los países socialistas" con Daniel Baggioni, D. Creissels y J. Legrand, "situaciones lingüísticas de los países colonizados y excolonizados" con D. Morsly y F.L. Prudent [...] Al 4 - La mesa redonda final se tituló "Epistemología de la sociolingüística". El texto fue traducido al español por el autor de este artículo. Dicha traducción permanece inédita.

5 - En Calvet, 1993b, se trata con detalle esta problemática. Es más, vale la pena citar la primera parte de la conclusión: « De nombreux chercheurs ont souligné l'échec de ceux qui ont essayé de donner une définition de la sociolinguistique. Mais la raison de cet échec est simple : dans tous les cas, ces auteurs tentaient de définir la sociolinguistique par rapport à la linguistique. Or c'est l'inverse au'il faut faire. Si l'on prend au sérieux laffirmation, assez largement acceptée, selon laquelle la langue est un fait (ou un produit) social, alors la linguistique ne peut être définie que comme létude de la communauté sociale sous son aspect linguistique. Et la sociolinguistique ne peut a son tour se définir que comme la linguistique. »(pág. 124). 
de la variedad de las lenguas, en la medida en que ésta no se consideraba ya en la dialectología ( $y$, en parte también con respecto a lo ya considerado en la dialectología), b) estudio del 'status' de diferentes tradiciones lingüísticas en una misma comunidad; c) estudio del grado de conocimiento y empleo de la lengua común por parte de los diferentes estratos socioculturales de una comunidad." (1981:8). Es interesante observar que en su libro La sociolinguistique ${ }^{6}$, el profesor L.-J. Calvet dice varias cosas interesantes que pueden ayudarnos a comprender la heterogeneidad de la sociolingüística (i.e. de su objeto de estudio) y cómo se ubicaría dentro de la historia de las ideas lingüísticas. En el primer capítulo del libro (titulado 'La lucha por una concepción social de la lengua'), el autor presenta el origen del conflicto cuya responsabilidad recae en dos autores. Saussure y Meillet. El primero es el defensor del paradigma de la langue, es decir el sistema autónomo y cerrado; el segundo es defensor del paradigma de la parole, entendida esta como el hecho social. Luego le siguen autores de la más diversa índole tales como los marxistas soviéticos, y los pensadores anglófonos como Bernstein, Bright y Labov.

Hacia el final del libro Calvet propone una alternativa para no caer en las trampas lexicales de la denominación y por ende de la definición. Dicha alternativa consiste en proponer que, "l'objet d'étude de la linguistique n'est pas seulement la langue ou les langues mais la communauté sociale sous son aspect linguistique. De ce point de vue, il n'y a plus lieu de distinguer entre sociolinguistique et linguistique, et encore moins entre sociolinguistique et sociologie $d u$ langage. " (1993b :109). Esta proposición no ha sido discutida, al menos en las diferentes publicaciones que versan sobre este tema. Pero lo que sí queda suficientemente claro es que podemos considerar la sociolingüística como un enfoque particular que intenta explicar la convergencia de variables tanto lingüísticas (entiéndase 'sistema') como socioculturales de una comunidad dada, en un acto comunicativo específico. Es por eso que el tema del bilingüismo como realidad social de una comunidad dada es uno de los tantos objetos de estudio de la disciplina mencionada. Y si a eso le añadimos que al interior de la sociolingüística hay una mirada más detallada que se ocupa del fenómeno en cuestión, esto es, del bilingüismo, entonces la perspectiva glotopolítica resultaría la más adecuada para hacerlo. La glotopolítica es una versión ampliada del enfoque politológico: políticas lingüísticas territoriales. Veamos, a grandes rasgos, la mirada glotopolítica.

\section{La Glotopolítica}

Hemos mencionado en el título de este trabajo que el fenómeno del bilingüismo va a ser estudiado desde la óptica 'glotopolítica'. En cada una de las partes de este documento, vamos a desglosar y explicar lo que quiere decir el término, por qué se utiliza y cuál es su justificación. Si bien lo glotopolítico tiene relación con las políticas lingüísticas y sus respectivas planificaciones $^{7}$, no se puede considerar exactamente como sinónimos. Es por eso que queremos citar inicialmente a J.B. Marcellesi (1986:33), quien fue uno de los gestores de dicho concepto, para que su declaración nos permita (re)iniciar el debate sobre la jerarquización de las lenguas habladas en una comunidad social:

D'abord les langues ne sont pas un objet [...] On doit les assumer dans leurs variations temporelles, spatiales, sociales. Il faut avoir à l'esprit qu'il y a une constante glottogenèse toujours à l'ouvre et que la seule naissance d'une langue est sa reconnaissance. La conception unifiante (ce qui ne veut pas dire simplement unificatrice) conduit nécessairement à des formes d'aliénation. Certes la planification linguistique se raidit devant la variation...Ce n'est pas une raison pour aligner sur elle la glottopolitique. D'autre part, sur le terrain, dans le temps ou dans la société, les langues ne sont pas souvent des réifications aux limites bien nettes. Ce sont des objets difficiles à compter (combien y a-t-il de langues romanes ?) si on voit leur existence comme des processus marqués par la dialectique de la satellisation vs la différentiation, l'identité linguistique étant un élément déterminé même s'il peut devenir et devient souvent ensuite surdéterminant si on prend pour la réalité ce qui $n^{\prime} e n$ est que l'ombre. $D$ ' où la nécessaire prise en compte, pour l'enseignement, de stratégies pluralistes et, quand il y a lieu, d'une saisie polynomique [...]

Este término fue forjado con especial cuidado por la Escuela de Rouen (Francia) bajo la dirección de J.-B. Marcellesi, L. Guespin y B. Gardin. Hablar de glotopolítica es pues hablar de un neologismo que está ligado necesariamente a la acción y que intenta por un lado, neutralizar la oposición entre lengua y habla determinando que toda manifestación lingüística es al tiempo individual y social, y por otro, designar las diferentes aproximaciones (enfoques) que una sociedad manifiesta en la acción sobre la lengua sea o no consciente de ello. A manera de ilustración veamos el caso de los que ha sucedido en Colombia a partir de la promulgación de la Constitución Política de 1991: se llevó a cabo una legislación sobre el papel del español frente a las lenguas indígenas y criollas con el fin de destacar en la realidad social (i.e. sociolingüística) colombiana, la coexistencia de varias lenguas pero manteniendo una postura política clara y definida en cuanto a la preeminencia del español como lengua no solo oficial sino también en varios casos, como lengua vehicular . Si bien "las lenguas y dialectos de los grupos étnicos son también oficiales en sus territorios", no siempre se ha cumplido a cabalidad esta proposición.

6 - Louis-Jean Calvet, La Sociolinguistique (Paris, Presses Universitaires de France, 1993b) El autor del presente artículo llevó a cabo la traducción al español de dicho texto. Lastimosamente permanece inédita por razones editoriales.

7 - Louis-Jean Calvet, La guerre des langues et les politiques linguistiques (Paris: Payot, 1987). Louis-Jean Calvet, La sociolinguistique (Paris: Presses Universitaires de France, 1993b). 
Esto quiere decir que cualquier decisión política que se tome sobre la(s) lengua(s) tiene necesariamente implicaciones prácticas que son de orden glotopolítico. La inevitable preeminencia del español sobre las demás lenguas que se hablan en el territorio colombiano nos muestra que el fenómeno plurilingüe se ve afectado por un hecho de diglosia, dando como resultado un fenómeno de monolingüismo vehiculars. El hecho de aceptar que el español sea tanto la lengua oficial como la lengua vehicular, es un acto glotopolítico.

La aplicación de este concepto puede verse con mayor claridad en la siguiente cita, tomada de Guespin y Marcellesi(1986, 15-16):

Le concept de glottopolitique rend compte d'un axe vertical liant le fait normatif ou antinormatif apparemment le plus insignifiant aux faits les plus saillants de politique de la langue. Il couvre aussi un terrain horizontal beaucoup plus vaste que celui que couvre la notion de politique de la langue, car la novation glottopolitique n'est pas toujours perçue en tant que telle : toute décision modifiant les rapports sociaux est, du point de vue du linguiste, une décision glottopolitique [...] La mesure n'es certes pas une décision de politique de la langue, mais elle comporte une importante incidence glottopolitique : elle concerne la mise en discours de l'économie, des rapports de production, du monde du travail. Toute mesure qui affecte la répartition sociale de la parole, même si son objectif n'est pas langagier, intéresse la situation glottopolitique. Le terme glottopolitique peut être utilisé à deux fins: à la fois pour l'évocation des pratiques et pour la désignation de l'analyse; la glottopolitique est donc à la fois une pratique sociale, à laquelle nul n'échappe (on "fait de la glottopolitique sans le savoir ", qu'on soit simple citoyen ou ministre de l'économie), et elle a vocation à devenir une discipline de recherche, une branche aujourd'huinécessaire de la sociolinguistique.

El marco de referencia que acabamos de esbozar nos brinda un panorama general del campo teórico en donde el fenómeno del 'bilingüismo' encuentra su asidero para poder ser explicado.

En nuestro referente colombiano abundan los comentarios superficiales sobre el fenómeno del bilingüismo. Dichos comentarios circulan libremente, convirtiéndose en una versión oficial de la realidad colombiana; son el fruto de una moda, de una información mal interpretada por parte de los medios de comunicación como de instancias superiores (p.ej. el Ministerio de Educación Nacional) y de una ausencia de análisis científico no sólo por parte de los sociolingüistas, quienes llevan a cabo su labor generalmente en las universidades, sino también por parte de los entes gubernamentales cuyo interés político desdibuja la armonía de la realidad social. Lo que sigue, sería un borrador para proponer un anteproyecto de investigación que apunta al diseño de una política lingüística equitativa en nuestro medio regional y nacional, con el fin de que los agentes 'décideurs' lleven a cabo una planificación acorde con nuestra realidad social. Queremos dejar en claro que no estamos en contra del inglés como lengua extranjera siempre y cuando la selección de esta lengua obedezca a un estudio previo de las necesidades sociolingüísticas en lengua materna (ya sea el español u otra lengua hablada en el territorio nacional), para poder equilibrar el valor de ser 'bilingüe' (hay que contar además con las actitudes emotivas ${ }^{9}$ de los hablantes).

El fenómeno del bilingüismo ha sido abordado en nuestro medio tan solo desde un enfoque glotopolítico interpretado, como lo acabamos de mencionar, de una manera que conviene a intereses particulares, obviando los referentes naturales inmediatos de la comunicación cultural colombiana. A más de esto, es visto desde una óptica restringida de la mecánica sociolingüística dejando de lado entre otras cosas, el enfoque psicolingüístico. Uno encuentra extraño que en el intento de hacer bilingües las regiones, cuestiones tales como los modelos teóricos de la lingüística cognitiva, la psicología del lenguaje, la ecología de las lenguas por solo mencionar algunos, estén ausentes en los proyectos que se socializan desde los entes "décideurs". Preguntas tales como ¿qué ventajas y desventajas cognitivas se encuentran en el fenómeno del bilingüismo?, ¿hay diferencias sociocognitivas entre individuos monolingües y bilingües (incluso plurilingües)?, ¿qué tipo de incidencia tienen variables sociolingüísticas como la edad, el sexo, el nivel educativo, la procedencia regional, el entorno inmediato y mediato en el aprendizaje de una nueva lengua?, ¿qué tipos de métodos son los más aconsejables cuando se trata de aprender una lengua en un medio exolingüe? No se aclara qué tipo de bilingüismo se lleva a cabo en cada región: compuesto y coordinado, simultáneo y secuenciado, temprano y tardío, aditivo y sustrayente, etc. Más aún, ¿qué papel juegan en el proceso de aprendizaje los estilos y ritmos cognitivos de los estudiantes?

Ahora bien, se ha asumido que el bilingüismo es la capacidad de hablar en otra lengua diferente de la materna con el propósito de ser "más competentes y comunicativos" en un mundo globalizado. Pero si bien

8 - Rafael Torres Quintero (2000:184) lo ilustra muy bien: "Lengua oficial es aquella que el gobierno de una nación impone constitucionalmente y en la que se difunden las leyes, se ejecuta la administración, se imparte la enseñanza y es reconocida por los usuarios como lengua de obligatorio aprendizaje aunque no sea para ellos la lengua materna o primera lengua aprendida en el hogar. El castellano es lengua oficial en todos los países de Hispanoamérica aunque no sea lengua materna para todos los que la hablan ni sea tampoco lengua vernácula concepto este que se aplica sólo a las de origen europeo." Véase además los Artículos 7,10,70, de la Constitución de 1991.

9 - Que en la teoría sociolingüística se definen como "actitudes positivas" y "actitudes negativas" Yolanda Lastra (2003:418) las presenta así: "Las actitudes hacia la lengua son cualquier índice afectivo, cognoscitivo o de comportamiento de reacciones hacia diferentes variedades de la lengua o hacia sus hablantes [...] La definición mentalista de actitud como estado neurológico y mental de predisposición indica que las actitudes no son observables sino que se tienen que inferir por introspección. En cambio la definición conductista localiza a las actitudes en el comportamiento mismo." 
es cierto que el Ministerio de Educación Nacional de Colombia justifica la política de bilingüismo en el país alegando: 1) la necesidad de formar individuos competentes en un mundo globalizado:

El Gobierno Nacional tiene el compromiso fundamental de crear las condiciones para que los colombianos desarrollen competencias comunicativas en otra lengua. Tener un buen nivel de inglés facilita el acceso a oportunidades laborales y educativas que ayudan a mejorar la calidad de vida. Ser competente en otra lengua es esencial en el mundo globalizado, el cual exige poderse comunicar mejor, abrir fronteras, comprender otros contextos, apropiar saberes y hacerlos circular, entender $y$ hacerse entender, enriquecerse y jugar un papel decisivo en el desarrollo del país. Ser bilingüe amplía las oportunidades para ser más competentes y competitivos. (MEN, 2006, Sección Carta Abierta)

y 2) la inclusión de elementos conceptuales que soportan una política lingüística artificial:

El bilingüismo se refiere a los diferentes grados de dominio con los que un individuo logra comunicarse en más de una lengua y una cultura. Estos diversos grados dependen del contexto en el cual se desenvuelve cada persona. Así pues, según el uso que se haga de otras lenguas distintas a la materna, éstas adquieren el carácter de segunda lengua o de lengua extranjera [...]

La lengua extranjera, en cambio, es aquella que no se habla en el ambiente inmediato y local, pues las condiciones sociales cotidianas no requieren su uso permanente para la comunicación. Una lengua extranjera se puede aprender principalmente en el aula y, por lo general, el estudiante está expuesto al idioma durante períodos controlados. A pesar de no ser usada en circunstancias diferentes a las académicas, los estudiantes de una lengua extranjera pueden alcanzar altos niveles de desempeño para ser comunicadores eficientes cuando así lo requieran. (MEN, 2006, 1. Las negrillas son nuestras),

Su objetivo apunta a dar una idea integral del fenómeno bilingüe, pero falla cuando en el ejercicio de aplicación cotidiano se hace lo contrario al ideal esperado. Esta actitud contraria obedece tal vez a que las variables psicosociolingüísticas han sido dejadas de lado en la constitución de una política lingüística 'bilingüe' para darle un énfasis exagerado al plano político / económico (el de la competitividad):

En el contexto colombiano y para los alcances de esta propuesta, el inglés tiene carácter de lengua extranjera. Dada su importancia como lengua universal, el Ministerio de Educación ha establecido dentro de su política mejorar la calidad de la enseñanza del inglés, permitiendo mejores niveles de desempeño en este idioma. Así pues, se pretende que los estudiantes al egresar del sistema escolar, logren un nivel de competencia en inglés B1 (Pre intermedio). (MEN, 2006, 1)

Con ello pretenden brindar un lenguaje común que permita a niños, niñas y jóvenes mayor acceso al mundo de hoy. Este hecho se ve confirmado por los datos suministrados por el Icfes respecto a las pruebas del 2004, según los cuales el noventa y nueve por ciento de los estudiantes seleccionaron el inglés en el examen de estado. (MEN, 2006, 3)

Y se establecen para ello, medidas de competencia (lingüístico/comunicativa) en lengua inglesa, siguiendo, como es de suponerse, estándares internacionales. De ahí la siguiente tabla ${ }^{10}$ :

\begin{tabular}{|c|c|c|c|}
\hline $\begin{array}{l}\text { NIVELES SEGÚN } \\
\text { EL MARCO EUROPEO }\end{array}$ & $\begin{array}{l}\text { NOMBRE COMÚN DEL } \\
\text { NIVEL EN COLOMBIA }\end{array}$ & $\begin{array}{l}\text { NIVEL EDUCATIVO EN EL QUE } \\
\text { SE ESPERA DESARROLLAR } \\
\text { CADA NIVEL DE LENGUA }\end{array}$ & $\begin{array}{l}\text { METAS PARA EL SECTOR } \\
\text { EDUCATIVO A } 2019\end{array}$ \\
\hline $\mathrm{A} 1$ & Principiante & Grados 1 a 3 & \\
\hline A2 & Básico & Grados 4 a 7 & \\
\hline B1 & Preintermedio & Grados 8 a 11 & $\begin{array}{l}\text { Nivel mínimo para el } 100 \% \\
\text { de los egresados de } \\
\text { Educación Media }\end{array}$ \\
\hline \multirow[t]{2}{*}{ B2 } & \multirow[t]{2}{*}{ Intermedio } & \multirow{4}{*}{ Educación Superior } & $\begin{array}{l}\text { Nivel mínimo para } \\
\text { docentes de inglés }\end{array}$ \\
\hline & & & $\begin{array}{c}\text { Nivel mínimo para } \\
\text { profesionales de otras carreras }\end{array}$ \\
\hline C1 & Preavanzado & & $\begin{array}{c}\text { Nivel mínimo para los nuevos } \\
\text { egresados de licenciatura } \\
\text { en idiomas }\end{array}$ \\
\hline $\mathrm{C} 2$ & Avanzado & & \\
\hline
\end{tabular}

10 - Tomada del MEN, 2006:2 
Es por eso que buscamos precisar las características del fenómeno bilingüe desde la óptica sociolingüística, intentando mostrar que se requiere de la consideración de diversas variables naturales de nuestra realidad social para poder formular una política lingüística eficaz. Es más, en la Declaración Universal de Derechos Lingüísticos ${ }^{11}$ el propósito de convivencia, respeto y consideración de las realidades lingüísticas naturales puede ofrecer mejores resultados en lo que denominan la Paz lingüística:

La Declaración es un texto necesario, tal como manifiestan sus Preliminares, para "corregir los desequilibrios lingüisticos de manera que asegure el respeto y el pleno desplegamiento de todas las lenguas y que establezca los principios de una paz lingüistica planetaria justa y equitativa, como factor principal de la convivencia social".

Y si esta paz ha de ser justa y equitativa es porque en los fundamentos de la Declaración está el principio básico de la igualdad de todos los pueblos y de todas las lenguas. Ni las características de los pueblos (económicas, sociales, religiosas, culturales, demográficas, etc.) ni las caracteristicas de las lenguas no justifican ningún tipo de discriminación; por tanto, todas las comunidades lingüisticas son sujetos de los mismos derechos ${ }^{12}$.

\section{Planteamiento del problema}

¿Qué tipo de elementos teóricos y metodológicos se requieren para poder diseñar una política lingüística acorde con la realidad social colombiana? Hay un considerable número de respuestas para intentar solucionar el interrogante. Todo depende del punto de vista que se considere y del objeto de estudio que se seleccione. No obstante, para este caso específico, cuando se habla del diseño de una política lingüística (como el resultado de una operación microlingüística), se está hablando también de una planificación lingüística, es decir de la puesta en marcha a través de una legislación que solo está en manos del Estado (operación macrosociolingüística). Consideramos que la respuesta se debe construir a partir del marco referencial mencionado más arriba:

sociolingüística $\longrightarrow$ glotopolítica bilingüismo.

La pregunta que planteamos supra, subyace al objeto de estudio propuesto en el esquema de análisis. Lo que buscamos inicialmente es analizar una tendencia glotopolítica en nuestro referente actual: La preferencia de una lengua en detrimento de otras por razones que no son lingüísticas sino sociales.

\section{Esquema de análisis}

1. Objeto: Implantación de una política bilingüe español/inglés en algunas regiones de Colombia. Aproximación glotopolítica.
2. Problema:

Presentación

Presupuestos

a) Cualquier propuesta, sea pública o privada que busque modificar la realidad social y lingüística de un territorio determinado, debe fundamentarse en un conocimiento sólido de su historia, sus tendencias, sus miembros, en síntesis, de su cultura.

b) El bilingüismo (i.e. plurilingüismo, multilingüismo) no es el resultado de una mera reglamentación oficial plasmada en documentos. Es más bien el producto de la convergencia de una realidad social natural (por ejemplo la coexistencia de hablantes de dos o más lenguas en un territorio) y una organización administrativa-política que permita su desarrollo libre y equitativo.

c) El bilingüismo ha sido un fenómeno ampliamente estudiado por la sociolingüística y es esta disciplina la que puede determinar los criterios teóricos y metodológicos para su adecuada comprensión a través de enfoques tales como el glotopolítico o el politológico.

d) La aproximación glotopolítica busca explicar la articulación de los procesos de selección políticos en el establecimiento de acuerdos de convivencia e intereses de los miembros de la comunidad.

e) El enfoque de la politología lingüística se ocupa de las intervenciones sobre las situaciones lingüísticas que son el fruto de un proyecto cultural (p.ej. la globalización) y determina los criterios más acertados para hacer gestión. Dicho enfoque se fundamenta en el concepto de 'modelo gravitacional' que se define, siguiendo a Calvet (2001), como una representación abstracta de relaciones concretas que se encarnan en un sitio dado, en una situación dada y a través de hablantes dados.

f) La sociolingüística aplicada a los problemas pedagógicos centra su atención en la manera como se puede organizar el lugar de una lengua -sea materna, segunda o extranjera- en los diferentes niveles educativos de una comunidad.

g) Las acciones in vivo $e$ in vitro deben entenderse como dos tipos de gestión del plurilingüismo. En otros términos, dichas acciones tienen que ver con las estrategias que los miembros de una comunidad deben trazar para lograr una comunicación no solo natural sino también efectiva. La gestión in vivo centra su atención en la manera cómo los hablantes, frente a un problema de comunicación, logran resolverlo; por ejemplo tenemos el caso de los pídgins que son un tipo de idiomas accesorios que responden a necesidades en extremo limitadas como por ejemplo el intercambio comercial. El pídgin es el resultado de una combinación morfolexical entre lenguas vernáculas con una lengua de prestigio mayor (español, francés, inglés).La gestión in vitro por el

11 - Comité de seguimiento de la Declaración Universal de los Derechos Lingüísticos. Declaración Universal de los Derechos Lingüísticos (Barcelona: Comité de seguimiento de la Declaración Universal de los Derechos Lingüísticos (Barcelona, 1998).

12 - Ibidem, págs. 12-13. 
contrario, es una acción preparada y estudiada por los especialistas en política lingüística para determinar el papel de las lenguas en una realidad social dada. Un ejemplo de ello es la propuesta de bilingüismo en nuestro país haciendo énfasis en la importancia del inglés como lengua extranjera vehicular de primer orden.

h) La propuesta para que Bogotá y Cundinamarca, Barranquilla, Cali, Medellín, Quindío, Bucaramanga y ahora el Huila (territorios en esencia monolingües como resultado de la imposición histórica del español $^{13}$ ) se constituyan en territorios bilingües español/inglés, se puede considerar tan solo como un esbozo de política lingüística incipiente. Se requiere de un análisis detallado de la realidad sociolingüística colombiana para poder determinar hasta qué punto es válido -y procedente- anteponer la política lingüística español+lengua extraterritorial (inglés) a la política español+lengua intraterritorial (cualquiera de las lenguas autóctonas de nuestro país, esto es las indígenas, las criollas, la de la comunidad Rom, la de la comunidad de sordos). Hay que conectar adecuadamente acción in vivo $y$ acción in vitro.

i) Detrás de la formulación de una política y una planificación lingüísticas, se encuentran aspectos sociolingüísticos tales como las actitudes sociolingüísticas (positivas y negativas), la realidad social, las lenguas en contacto, el mercado lingüístico, las redes sociales y las lenguas, los diferentes niveles de bilingüismo, el cambio lingüístico en marcha, los ideologemas, etc.

j) Tal como lo menciona el Observatorio Europeo de Plurilingüismo, cada lengua de cultura opera una apertura específica sobre el universo humano. Defender y promover las diferentes lenguas, es defender y promover una parte de la universalidad, de lo humano.

k) Según lo manifiesta la Declaración Universal de los Derechos Lingüísticos, articular los derechos lingüísticos de comunidades, grupos y personas que comparten un mismo espacio es imprescindible para garantizar la convivencia, pero resulta extraordinariamente complejo. Por ello la Declaración tiene en cuenta los derechos de las comunidades lingüísticas asentadas históricamente en su territorio con el fin de establecer una gradación, aplicable en cada caso, de los derechos de los grupos lingüísticos con diferentes grados de historicidad y de autoidentificación, y de los individuos que viven fuera de su comunidad de origen.

l) Si bien es cierto que el inglés ha adquirido el estatus de lengua global vehicular (o de servicio) de manera natural, esto no quiere decir que en una planificación lingüística in vitro (acorde con la realidad social in vivo de un territorio) se excluyan de forma arbitraria otras lenguas que son igualmente poseedoras de una amplia tradición cultural.

m) La lectura (i.e. interpretación) que se ha hecho de los documentos oficiales tales como la Consti- tución Política de 1991, la Ley 115 o Ley General de Educación, el Marco Europeo de Lenguas, los Estándares Básicos de Competencias en Lenguas Extranjeras: Inglés, la política de protección de las lenguas de los grupos étnicos presentes en el territorio colombiano, la Ley 1381 del 25 de enero de 2010 conocida también como la Ley de Lenguas, así como de estudios científicos del área de la lingüística entre otros, no ha sido coherente $y$ dialógica con la realidad social colombiana.

n) Resultaría conveniente indagar cómo la propuesta de implementación del bilingüismo, como un acto de gestión in vitro, permitiría la constitución de una cultura ciudadana integral y auténtica.

o) Cuando se ha abordado el fenómeno del bilingüismo en nuestro país se ha hecho un énfasis en la lengua extranjera que se debe seleccionar y trabajar (el inglés en nuestro caso), pero se ignora el tratamiento de la lengua materna, el español. Esta actitud niega de entrada el hecho bilingüe. No hay que negar además que un buen monolingüismo es una condición del plurilingüismo. A más de esto, hay que considerar los niveles de desempeño en lengua materna como una condición sine qua non para el trabajo con la lengua extranjera. Ya lo menciona el profesor Miguel de Zubiria: "Con estos resultados [onerosos, negativos, MM], es un despropósito que un país en el que los estudiantes no alcanzan los mínimos niveles de comprensión lectora en su lengua materna, se pretenda abordar el desarrollo de las competencias interpretativas en una segunda lengua."

p) Se debe proponer también un modelo glotopolítico para el español con el fin de cubrir todos los frentes culturales y donde entre en un proceso de diálogo cultural con aquellas lenguas autóctonas que ocupan igualmente un espacio importante en nuestro contexto colombiano: maternas, segundas como es el caso de las lenguas indígenas, criollas, la de la comunidad Rom y la de la comunidad de sordos.

q) Hay tres categorías de factores que permiten formular políticas lingüísticas 'reales': (i) Aquellas que constituyen una fotografía de la situación actual y vigente de una comunidad lingüística; (ii) Las que podrían constituir eventualmente una explicación de esta situación y que serían las causas cuyos factores precedentes mostrarían los efectos. Claro está que podrían causar algunos inconvenientes glotopolíticos; por ejemplo, iel poder económico de un país es directamente proporcional al peso de su lengua oficial?; (iii) Están los factores que permitirían hacerse una idea de esta situación. Por ejemplo el crecimiento demográfico de un país tendría una incidencia notable en el número de hablantes de la(s) lengua(s) de ese país.

r) Lo mencionado en los anteriores presupuestos nos muestra que el cultivo del bilingüismo (español/lengua autóctona [criolla/indígena], español/lengua extranjera [alemán, chino, francés, inglés, italiano, japonés, portugués, etc.]), solo es

13 - Glotofagia exitosa. Para más detalles sobre este concepto, véase Calvet 1974. 
posible en Colombia si los parámetros de estatus, valor, situación, contexto, motivación y necesidades institucionales (i.e. culturales) son tenidos en cuenta. Cualquier otro intento, como el del más reciente proyecto gubernamental Colombia Very Well, fracasará por su artificialidad y por ende de su inaplicabilidad. Y los cambios afanosos de denominación pretendiendo darle un nuevo rumbo a la dinámica bilingüe.

\section{Interrogantes}

a) ¿Cómo está constituida la realidad sociolingüística colombiana?

b) ¿Qué tipo de niveles de bilingüismo se presentan en Colombia?

c) ¿Qué elementos se deben tener en cuenta para formular (i.e. diseñar) una política y una planificación lingüísticas (i.e. un espacio glotopolítico) que sean viables para el desarrollo multicultural de nuestro país, teniendo en cuenta la realidad sociolingüística colombiana?

\section{Justificación del problema}

\section{Estado de la cuestión}

La pregunta por el estado del bilingüismo en Colombia se inscribe inicialmente en las propuestas de la etnolingüística que se centran en la relación entre el español como lengua oficial dominante y las lenguas indígenas y criollas como lenguas igualmente oficiales pero sometidas políticamente (Diglosia). Es en este dominio en donde se encuentra el mayor número de trabajos. En razón a que la realidad social colombiana constituye la coexistencia natural de hablantes de aproximadamente 64 lenguas indígenas ${ }^{14}, 2$ lenguas criollas (una de base española, el 'palenquero' y la otra de base inglesa, el 'criollo sanandresano') y el español la atención se ha concentrado en esta peculiaridad natural que ha desembocado en la propuesta de políticas lingüísticas incipientes ${ }^{15}$. Por el contrario no son tan numerosos los estudios consagrados a la problemática sociolingüística del bilingüismo español/lengua extranjera de prestigio (alemán, francés, inglés, italiano, portugués, etc.) en nuestro medio. Los trabajos consagrados a este tipo de bilingüismo proceden de la didáctica de la lengua extranjera en donde los postulados sociolingüísticos son gentilmente parafraseados. Hay también trabajos que se ocupan de la relación español/lengua extranjera (con mayor atención en el inglés) en cuanto a procesos de calco semántico y de la traducción. Las limitaciones de los anteriores enfoques radican en el hecho de que no se ha ilustrado suficientemente la realidad sociolingüística colombiana en donde los hablantes nativos de las lenguas indígenas y las lenguas criollas han debido -por razones glotopolíticas- apoyarse en el español no solo como lengua vehicular, sino también, en caso extremo, como lengua de cultura. De esta forma, estos hablantes no han constituido un espacio lo suficientemente marcado para 'nacionalizar' su visión del mundo. Hay un marcado caso de diglosia y en cierta manera una tendencia a la glotofagia. De igual forma, el querer implantar a manera de gestión in vitro políticas culturales carentes de una sólida fundamentación científica, que privilegian, entre otras cosas, un idioma extranjero (el inglés), en detrimento de otras lenguas (autóctonas [lenguas indígenas] y extranjeras como las ya citadas) es lo que ha impedido consolidar una política y una planificación lingüísticas acordes con la realidad social colombiana (proceso glotopolítico en marcha)

\section{Importancia del problema}

Resulta conveniente implementar las estrategias sociolingüísticas (i.e. glotopolíticas) que permitan el desarrollo equitativo de las lenguas que forman parte de la realidad social cotidiana colombiana. Es por eso que se requiere de una política cultural contundente. Una vez llevado a cabo este proceso se puede pensar en el intercambio plurilingüe con comunidades de otros países que cuentan con una realidad social diferente a la nuestra. Esto es lo que se ajustaría al plan propuesto inicialmente por la comunidad europea y que se podría conectar con la realidad latinoamericana.

\section{Marco teórico}

Se asume como marco teórico las propuestas de LouisJean Calvet (1993b-2000-2002) y de Claude Hagège $(1996,2012)$ en lo concerniente a la (re)consideración del bilingüismo y las políticas culturales (lingüísticas y educativas) que se deben implementar teniendo en cuenta la realidad social de los territorios. Ahora bien, las propuestas de estos autores si bien han sido gestadas para las realidades de Europa, África y algunos lugares de Asia, sirven como base para propuestas en el medio latinoamericano y en especial, para el caso colombiano. A más de esto vamos a considerar algunas reflexiones teóricas que han propuesto estudiosos colombianos sobre el fenómeno del bilingüismo y su correspondiente implementación como las reflexiones del profesor Armando Rico en el ámbito de la sociolingüística colombiana (1980-1981 y 1998) así como en el ámbito de la enseñanza de lenguas (1996). De igual forma, las reflexiones de

14 - Frente a esta aproximación dice Patiño (1991:148): "Esta es una apreciación aproximada. No es posible presentar una cifra más precisa y realista mientras las investigaciones etnolingüísticas no aclaren, en muchos casos, si determinadas variedades deben considerarse como lenguas o como dialectos."

15 - Que generan por ejemplo políticas educativas. Estas se apoyan, entre otras cosas, en el Artículo 10 de la Constitución Política de Colombia: "Idiomas. El castellano es el idioma oficial de Colombia. Las lenguas y dialectos de los grupos étnicos son también oficiales en sus territorios. La enseñanza que se imparta en las comunidades con tradiciones lingüísticas propias será bilingüe." (El destacado es nuestro). Para más detalles sobre estos aspectos véase Carlos Patiño Rosselli (1991:145-207), así como el libro Lenguas Amerindias. Condiciones sociolingüísticas en Colombia, 1997. 
Oakley Forbes $(1987,1989,2005)$, de Anne-Marie de Mejía y Lionel Tovar (1999 y 2002). Como complemento, nos apoyaremos en la propuesta del profesor Orlando Fals Borda en cuanto al "Principio de endogénesis contextual" que busca un cambio en la visión frente al colonialismo intelectual que nos ha dominado (Fals Borda, 1981 y 2003).

\section{Solución}

\section{Para el interrogante a):}

Hipótesis: Colombia está constituida por un mosaico cultural fruto de la convivencia de hablantes nativos del español, de las lenguas indígenas, de las lenguas criollas, de la comunidad $\operatorname{Rom}^{16}$, de los usuarios de la lengua de señas y en un porcentaje menor, de lenguas extranjeras.

Sustentación: La realidad lingüística colombiana es en esencia multilingüe y por ende multicultural. En el territorio colombiano, un alto porcentaje, por no decir la mayoría, tiene el español como lengua materna y son monolingües. De la misma forma, hay hablantes bilingües naturales cuya lengua materna puede ser una lengua indígena o una lengua criolla y que tienen como segunda lengua el español ${ }^{17}$. Encontramos hablantes nativos de lengua española que manejan una lengua extranjera de prestigio como el alemán, el francés, el inglés, el italiano o el portugués. De igual forma, contamos con hablantes nativos de estas lenguas de prestigio que se han radicado en Colombia y cuyo manejo del español se hace necesario para convivir en el territorio nacional. Si bien es cierto que el multilingüismo hace parte de la realidad social colombiana, es conveniente decir también que dicho fenómeno no tiene un peso fuerte en la cotidianidad glotopolítica del país. Es en español que se vehicula el trasegar nacional; las lenguas indígenas y criollas no han dejado de ser elementos exóticos que se les mira con indiferencia e incluso ignorancia. No sobra decir que hay un marcado caso de diglosia.

\section{Para el interrogante b):}

Hipótesis: En Colombia encontramos tres niveles de bilingüismo: un primer nivel es el 'natural' en donde encontramos los hablantes que utilizan en su cotidianidad una lengua indígena o criolla como lengua materna y el español como una lengua vehicular (segunda). Otro nivel es el de los hablantes que tienen el español como lengua materna pero que han aprendido una lengua extranjera de prestigio ya sea en colegios bilingües, en la universidad o en viajes al exterior. El tercer nivel, más restringido es el de los hablantes que son hijos de parejas bilingües y que encuentran en el hogar un espacio bilingüe auténtico.

Sustentación: Los tres niveles descritos son una mera aproximación a la realidad sociolingüística colom- biana. Es probable que haya otras propuestas de clasificación. Lo que si es cierto es que Colombia es un país multilingüe en donde de acuerdo a los contextos de comunicación y a las imposiciones glotopolíticas, se escoge una o varias lenguas para llevar a cabo el intercambio lingüústico. Si bien el español es la lengua oficial en todo el territorio nacional y su utilización es completamente natural, hay espacios restringidos en donde otras lenguas se privilegian por cuestiones culturales. Este fenómeno es conocido como dominio sociolingüístico en donde la organización social es la base de la descripción de las elecciones lingüísticas. Claro está que no solo los elementos sociales aportan una cuota valiosa, los elementos psicológicos son definitivos para integrar el proceso de elección lingüística.

\section{Para el interrogante c):}

Hipótesis 1: Se deben tener en cuenta grosso modo, tres elementos: 1) Examen de la realidad social, 2) toma de decisiones (planeación propiamente dicha), 3) implementación. Como punto alternativo se propone una evaluación.

Sustentación 1: Se presentará, de manera esquemática, las etapas de la planeación lingüística siguiendo lo propuesto por Tovar (1999: 223-238), con el fin de aplicarlas a datos sociolingüísticos previamente establecidos:

\section{Examen de la realidad}

1.1. Inventario de necesidades

1.1.1. de los planificadores

1.1.2. de la comunidad objetivo

1.2. Análisis de factores sociales

1.2.1. sociodemográficos

1.2.2. políticos

1.2.3. lingüísticos

1.2.4. sociopsicológicos

1.2.5. religiosos

1.2.6. socioeconómicos

1.2.7. económicos

1.3. Factores individuales

1.3.1. la edad

1.3.2. el desarrollo de la lengua materna

1.3.3. la adquisición previa de otra segunda lengua

1.3.4. la aptitud

1.3.5. la motivación

1.3.6. la actitud

1.3.7. la personalidad

1.3.8. los estilos cognitivos

1.3.9. la especialización hemisférica

1.3.10. las estrategias de aprendizaje

1.3.11. la memoria

1.3.12. la conciencia de estar adquiriendo otra lengua

1.3.13. la persistencia

1.3.14. el interés

16 - Para el caso de la comunidad sanandresana, hay una tercera lengua, el inglés estándar caribeño. No queremos entrar en detalle en cuanto a particularidades sociolingüísticas de los grupos indígenas, por ejemplo, las comunidades indígenas del Vaupés. Para más detalles sobre este último caso, véase François Correa 1997:443-492.

17 - No estamos ignorando el nivel de bilingüismo de la comunidad de sordos. No obstante, nuestro interés apunta a las lenguas orales. 
1.3.15. los problemas de aprendizaje y del lenguaje

2. Toma de decisiones (Planeación propiamente dicha)

2.1. Determinación de objetivos

2.2. Codificación

2.2.1. afectiva

2.2.2. mecánica

2.3. Elaboración

3. Implementación

3.1. Organización

3.2. Interdisciplinariedad

4. Evaluación

Estos elementos deben ser tenidos en cuenta para la formulación de una política lingüística acorde con la realidad social colombiana. Al mismo tiempo, podemos configurar el anteproyecto de investigación recopilando información y analizando dato por dato, los momentos de la planeación lingüística que llevarían a cabo las instancias del poder, i.e. el Estado colombiano.

Hipótesis 2: De igual forma, se puede llevar a cabo, de manera paralela, el proceso de evaluación de la política lingüística -en nuestro caso la formulada en el PNB- , siguiendo lo propuesto por Blanchet (2008), en donde se realicen evaluaciones diagnósticas, de seguimiento, de control de objetivos y de finalidades, de regulación de la heterogeneidad sociolingüística, etc., que permiten articular una política lingüística más cercana a las necesidades comunicativas de los miembros de la comunidad.

Sustentación 2: Según Blanchet 2009, la evaluación de la política lingüística está conformada por cinco puntos:

Evaluación de la situación sociolingüística de partida; Evaluación a priori de la pertinencia de la situación sociolingüística de llegada que se ha planeado;

Evaluación de la puesta en marcha de las intervenciones decididas;

Evaluación de los efectos y de los cambios provocados; Evaluación a posteriori de la nueva situación, es decir la situación de llegada.

El autor organiza su propuesta en un esquema de trabajo en donde justifica el término de aménagement linguistique y su pertinencia para la evaluación glotopolítica apoyándose en la propuesta de D. de Robillard:

Ainsi, "aménagement" constitue un concept englobant qui désigne une forme conscientisée, scientificisée et professionnalisée d'interventions visant à la modification des langues en ce qui concerne leur statut et leur corpus (Robillard, 1997 : 36), ce que l'auteur représente sous le schéma suivant (Robillard, 1997:39):

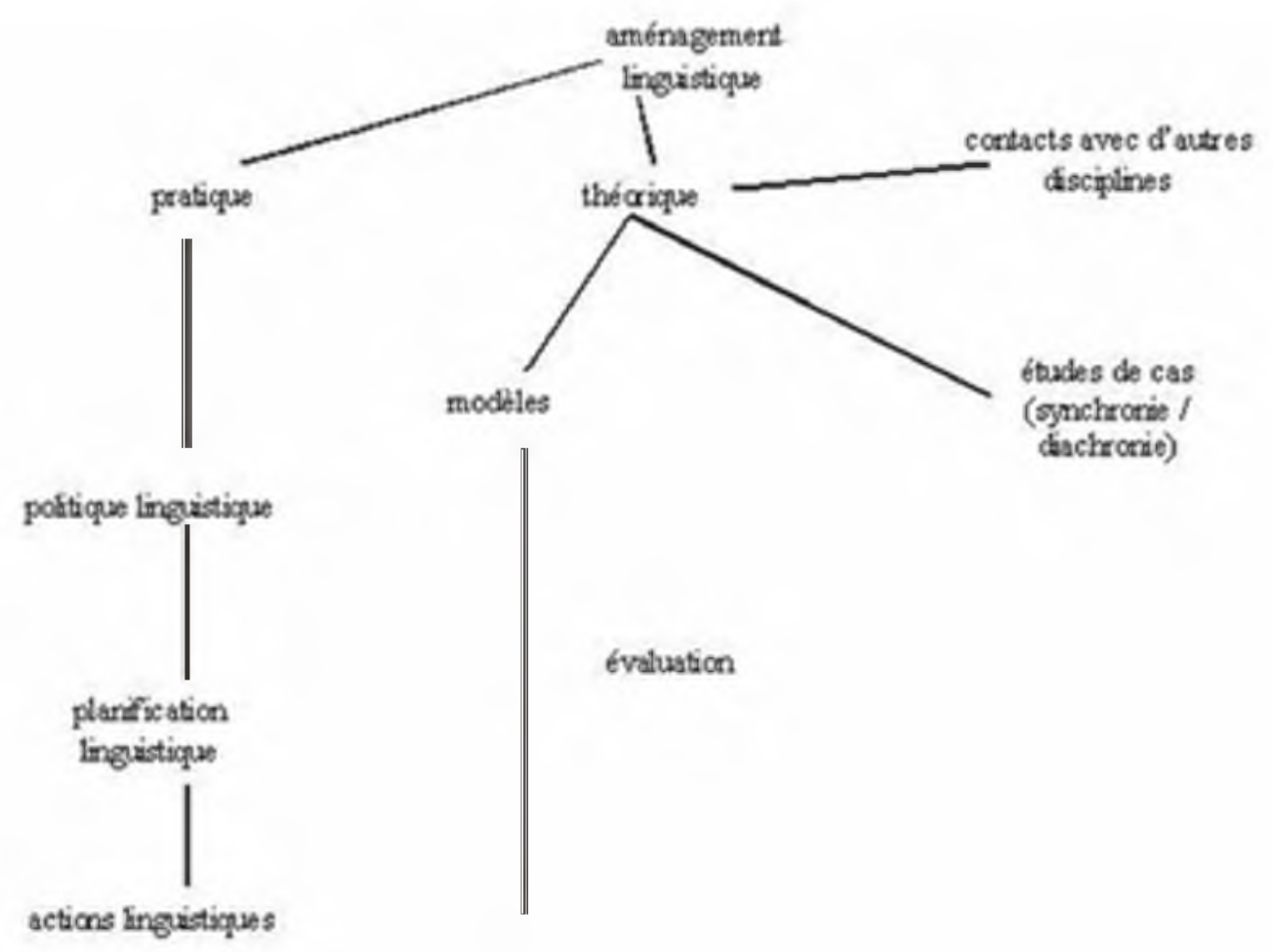

Fuente: Tomado de Blanchet, 2008 


\section{Conclusiones provisionales (a manera de Proyección)}

Hemos elaborado una semblanza teórica/ metodológica alrededor del bilingüismo, de las políticas lingüísticas y de la visión glotopolítica. Queda pendiente por desarrollar el anteproyecto de investigación, siguiendo inicialmente la propuesta de Tovar (1999) y enriqueciễndola con la propuestas de Mejía (2006), Hagễge $(1996,2012)$, Blanchet y las emanadas por el Ministerio de Educación Nacional a partir de 1991. Lo mencionado aquí es tan solo una suerte de ilustración y por ende de justificación de un referente sociolingüístico de nuestro país que debe orientarse hacia la integración cultural y no hacia la dispersión. Algunas de las ideas insinuadas han sido tratadas en los cursos de sociolingüística para los estudiantes del programa de lengua extranjera, en los cursos de lingüística para los estudiantes del programa de lengua castellana que he orientado así como en un proyecto de semillero de investigación. Quisiera añadir finalmente que este documento es una invitación a todos aquellos que están interesados y por ende involucrados en las actividades de la política nacional de bilingüismo adelantada desde el Ministerio de Educación Nacional, las gobernaciones, las alcaldías y por supuesto, las universidades, para unir fuerzas en la formulación de una política lingüística acorde con la realidad sociolingüística de Colombia.

\section{Referencias}

Blanchet, Philippe, 2007, "Quels 'linguistes' parlent de quoi, à qui, quand, comment et pourquoi? Pour un débat épistémologique sur l'étude des phénomènes linguistiques", in Blanchet, Philippe, Calvet, Louis-Jean, Robillard Didier de, Un siècle après le Cours de Saussure, la linguistique en question, Camets de l'Atelier de Sociolinguistique ${ }^{\circ}$ 1, Paris, L'Harmattan, p. 229-294.

Blanchet, Philippe, 2008, "La nécessaire évaluation des politiques linguistiques entre complexité, relativité et significativité des indicateurs", Les Cahiers du GEPE, N ${ }^{\circ} 1 / 2008$. L'analyse des pratiques d'évaluation des politiques linguistiques : une entrée pour l'étude des politiques linguistiques.

Pour le lire: http://www.cahiersdugepe.fr/index898.php

Bright, William. "Las dimensiones de la sociolingüística", Antología de estudios de etnolingüistica y sociolingüística. Editado por Pau Garvin y Yolanda Lastra. México D.F.: Universidad Nacional Autónoma de México, 1974.

Calvet, Louis-Jean. "La sociolinguistique et la ville: hasard ou nécessité", Marges linguistiques (Mai, 2002): 46-53.

Calvet, Louis-Jean. Sociolinguistique. Paris: Presses Universitaires de France, $1993 \mathrm{~b}$

Calvet, Louis-Jean. La guerre des langues et les politiques linguistiques. Paris : Payot, 1987.

Calvet, Louis-Jean. Linguistique et colonialisme. Petit traité de glottophagie. Paris: Payot, 1974.

Comité de seguimiento de la Declaración Universal de los Derechos Lingüísticos. Declaración Universal de los Derechos Lingüís ticos, Barcelona: Comité de seguimiento de la Declaración
Universal de los Derechos Lingüísticos Barcelona, 1998.

Correa, Francois. "Identidad social y ejercicio lingüístico en la región del Vaupés colombiano". Lenguas amerindias. Condiciones sociolingüísticas en Colombia. Bogotá: Instituto Caro y Cuervo, 1997.

Coseriu, Eugenio. "La socio- y la etnolingüística: sus fundamentos tareas". Anuario de Letras XIX (1981): 5-30.

Coste, Daniel. "Quelles évaluations pour quelles politiques linguistiques" In Les Cahiers du GEPE, No 1/2008. Online www.cahiersdugepe.fr/index696.php

Forbes, Oakley. "Recreolización y decreolización en el habla de San Andrés y Providencia". GlottaVol. II, No 2 (1987):13-17.

Forbes, Oakley. "Multiculturalismo y multilingüismo" www.fundacionbat.com.co/Multiculturalismoymultilinguismo.p df.

Gardin, Bernard, Jean-Baptiste Marcellesi et GRECO Rouen, éds. Sociolinguistique: Approches, théories, pratiques. Paris: Publications de l'Université de Rouen et PUF (2 vol.), 1980.

Guespin, L. et J.B. Marcellesi. " Pour la glottopolitique ", Langages 83 (1986):5-34.

Hagège, Claude. Contre Ia pensée unique. Paris : Editions Odile Jacob, 2012.

Hagège, Claude. L'enfant aux deux Iangues. Paris: Editions Odile Jacob, 1996.

Lastra, Yolanda. Sociolingüística para hispanoamericanos: una introducción. México, D.F.: El Colegio de México, 2003.

Marcellesi, Jean-Baptiste et Bernard Gardin. Introduction à lo sociolinguistique. La linguistique sociale. Paris : Larousse, 1974.

Meillet, Antoine. "Comment les mots changent de sens "Linguistique historique, linguistique générale. Paris: Champion, 1965.

Mejía, Anne-Marie, López, Alexis \& Peña, Beatriz (compiladores) Bilingüismo en el contexto colombiano. Iniciativas y perspectivas en el siglo XXI. Bogotá: Universidad de los Andes, Centro de Investigación y Formación en Educación, 2011.

Mejía, Anne-Marie de y Laura Fonseca. Orientaciones para políticas bilingües y multilingües en lenguas extranjeras en Colombia. Bogotá: Universidad de los Andes, 2008.

Ministerio de Educación Nacional. Programa Nacional de bilingüismo 2006. Bogotá: Ministerio de Educación Nacional, 2006.

Pardo, Felipe y Julia Baquero. "La estructura argumentativa: base para la comprensión y producción de textos científicos y argumentativos", Forma y Función 14 (2001): 98-118.

Pardo, Felipe y Julia Baquero. "Un ejercicio de análisis de texto científico", Forma y Función 3 (1988): 33-57.

Patiño Rosselli, Carlos. "Español, lenguas indígenas y lenguas criollas en Colombia", EI español de América hacia el siglo XXI. Bogotá: Instituto Caro y Cuervo, 1991.

Rico Ocampo, Armando. Enseñanza y aprendizaje de lenguas extranjeras en primaria. Una ventana al Multilingüismo y a lo Pluricultural. Armenia: Universidad del Quindío, 1998.

Rico Ocampo, Armando. Problèmes sociolinguistiques de la société colombienne. Mémoire de Maitrise, Université de Grenoble, 1981.

Robillard Didier de, 1997, articles "Action linguistique" (20), "Aménagement linguistique" (36-41), "Corpus" (102), "évaluation" (151-152), "Planification" (228-229), "Politique linguistique" (229230), "Statut" (269-270), in Moreau, Marie-Louise (éd.), Sociolinguistique, concepts de base, Sprimont (B), Mardaga.

Rona, José Pedro. "La concepción estructural de la sociolingüística" Antología de estudios de etnolingüística y sociolingüística. Editado por Paul Garvin y Yolanda Lastra. México D.F.: Universidad Nacional Autónoma de México, 1974.

Torres Quintero, Rafael. "La planeación lingüística", Obras Tomo II, Lingüística y Literatura. Bogotá: Instituto Caro y Cuervo, 2000.

Tovar, Lionel. "Bases para una verdadera planificación lingüística en Colombia", Perspectivas recientes del bilingüismo y de la educación bilingüe en Colombia. Editado por Anne-Marie de Mejía y Lionel Tovar. Cali: Universidad del Valle, 1999.

Truchot, Claude. 2008. L'analyse des pratiques d'évaluation des politiques linguistiques: un objet d'étude à constituer. http://cahiersdugepe.misha.frindex66i.php 